\title{
Plasma deconvolution identifies broadly neutralizing antibodies associated with hepatitis $C$ virus clearance
}

\author{
Valerie J. Kinchen, ${ }^{1}$ Guido Massaccesi, ${ }^{1}$ Andrew I. Flyak,, ${ }^{2}$ Madeleine C. Mankowski, ${ }^{1}$ Michelle D. Colbert, ${ }^{1}$ William O. Osburn, ${ }^{1}$ \\ Stuart C. Ray, ${ }^{1,3}$ Andrea L. Cox, ${ }^{1,3}$ James E. Crowe Jr, ${ }^{4,5,6}$ and Justin R. Bailey ${ }^{1}$ \\ 'Department of Medicine, Johns Hopkins University School of Medicine, Baltimore, Maryland, USA. Division of Biology and Biological Engineering, California Institute of Technology, Pasadena, California, \\ USA. ${ }^{3}$ Department of Oncology, Johns Hopkins University School of Medicine, Baltimore, Maryland, USA. ${ }^{4}$ Department of Pediatrics, Vanderbilt University Medical Center, ${ }^{5}$ Department of Pathology, \\ Microbiology, and Immunology, and ${ }^{6}$ anderbilt Vaccine Center, Vanderbilt University Medical Center, Vanderbilt University, Nashville, Tennessee, USA.
}

\begin{abstract}
A vaccine for hepatitis C virus (HCV) is urgently needed. Development of broadly neutralizing plasma antibodies during acute infection is associated with HCV clearance, but the viral epitopes of these plasma antibodies are unknown. Identifying these epitopes could define the specificity and function of neutralizing antibodies (NAbs) that should be induced by a vaccine. Here, we present the development and application of a high-throughput method that deconvolutes polyclonal anti-HCV NAbs in plasma, delineating the epitope specificities of anti-HCV NAbs in acute-infection plasma of $\mathbf{4 4}$ humans with subsequent clearance or persistence of HCV. Remarkably, we identified multiple broadly neutralizing antibody combinations that were associated with greater plasma neutralizing breadth and with HCV clearance. These studies have the potential to inform new strategies for vaccine development by identifying broadly neutralizing antibody combinations in plasma associated with the natural clearance of HCV, while also providing a high-throughput assay that could identify these responses after vaccination trials.
\end{abstract}

\section{Introduction}

A vaccine to prevent hepatitis $\mathrm{C}$ virus (HCV) infection is urgently needed. Although recently developed direct-acting antivirals are highly effective for HCV treatment, most individuals are unaware of their infection status, so they do not seek treatment and may continue to expose others $(1,2)$. Most infected persons do not have access to direct-acting antivirals, and treatment does not prevent reinfection after cure (3-7). Unfortunately, HCV vaccine development has been hampered by incomplete understanding of correlates of protective immunity in humans $(8,9)$ and by inadequate methods for assessing antibody responses induced by candidate vaccines.

Around $25 \%$ of individuals who are acutely infected with HCV spontaneously clear the infection without treatment (10). We and others have shown that this spontaneous clearance is associated with early development of broadly neutralizing plasma, suggesting that identifying neutralizing antibodies (NAbs) present in the plasma of these individuals could elucidate the NAbs that should be induced by a vaccine $(11,12)$. However, the epitope specificities of NAbs present in broadly neutralizing plasma are unknown. This plasma might contain NAbs similar to known broadly neutralizing monoclonal antibodies (bNmAbs), broadly neutralizing antibodies (bNAbs) with novel epitopes, or a diverse array of strain-specific NAbs.

Conflict of interest: AIF, JEC, and JRB are inventors of International Patent Application, Serial no. PCT/US2019/029315, pertaining to some of the antibodies presented in this article. JEC has served as a consultant for Takeda Vaccines, Sanofi Pasteur, Pfizer, and Novavax, is on the Scientific Advisory Boards of CompuVax, GigaGen, Meissa Vaccines, PaxVax, and is Founder of IDBiologics, Inc. Copyright: @ 2019, American Society for Clinical Investigation. Submitted: May 30, 2019; Accepted: August 1, 2019; Published: September 30, 2019. Reference information: J Clin Invest. 2019;129(11):4786-4796. https://doi.org/10.1172/JCI130720.
Available methods used to identify the epitopes targeted by polyclonal serum-neutralizing antibodies have notable limitations. One such method is isolating monoclonal antibodies (mAbs) from B cells. We and others have isolated bNmAbs from the B cells of individuals who cleared HCV infection (13-17), and we demonstrated that bNmAbs designated HEPC3 and HEPC74, which target the front layer of envelope protein E2 (E2), contributed to viral clearance in 2 individuals by driving the infecting virus to an unfit state (18). However, these bNmAbs may not fully represent the complete repertoire of polyclonal NAbs present in the plasma of these subjects before viral clearance, and these methods remain relatively labor intensive and time consuming, making it difficult to characterize a large number of subjects. One alternative approach is measurement of serum antibody binding to peptide arrays, but many NAbs bind to discontinuous, conformational epitopes rather than linear peptides $(9,19,20)$. A third approach is measurement of envelope protein 1 and 2-binding (E1/E2-binding) competition between serum antibodies and reference mAbs (21), but these assays are confounded by the abundance of E1/E2 binding but nonneutralizing antibodies in plasma. These nonneutralizing antibodies may compete with bNmAbs for overlapping or adjacent E1/E2 epitopes (22-27), so binding competition may not discriminate reliably between neutralizing and nonneutralizing antibodies in plasma. Given these limitations, previous studies have not comprehensively identified NAbs contributing to neutralizing breadth of plasma or NAbs that may be associated with clearance or persistence of HCV infection.

In this article, we present the development and application of a high-throughput method that delineates the epitope specificities of anti-HCV NAbs in polyclonal human plasma. Using this method, we show that the majority of anti-HCV plasma neutralizing activity 


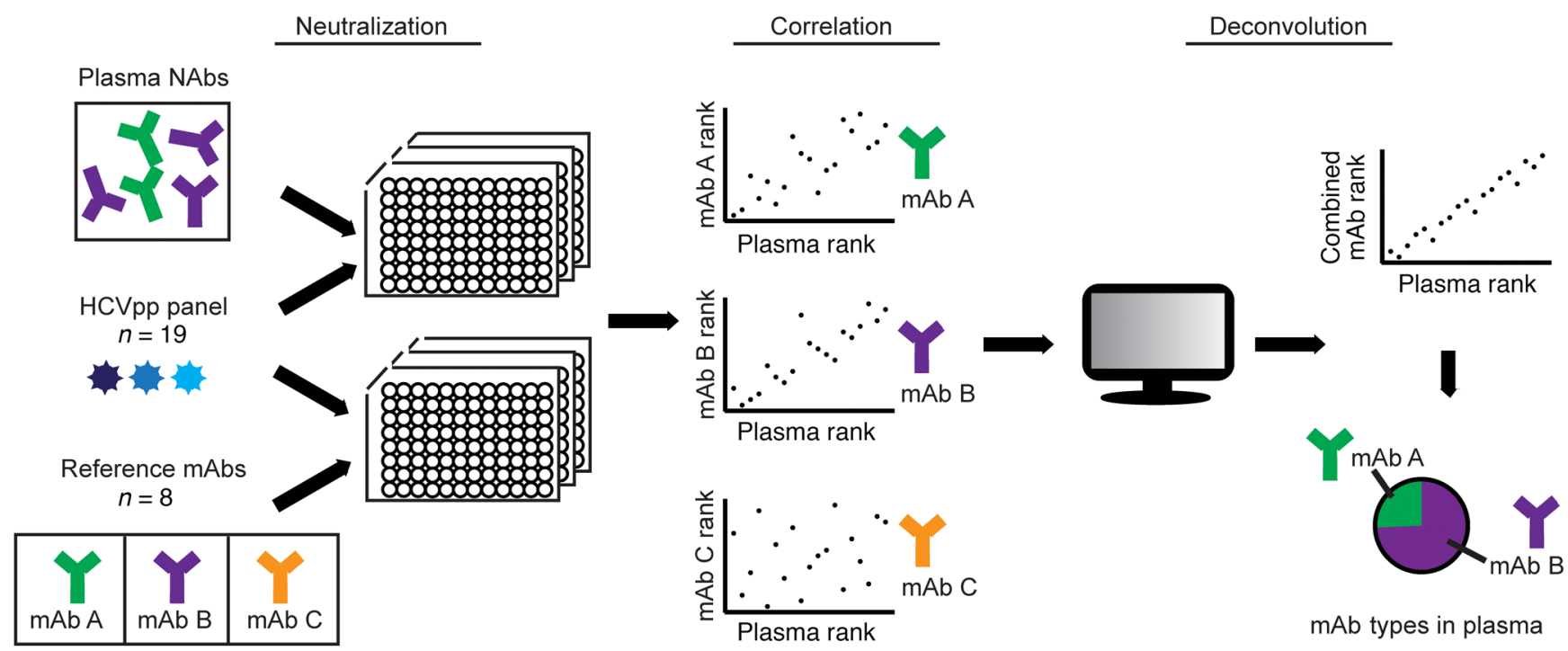

Figure 1. Deconvolution method. Neutralization of a panel of 19 HCVpp by 8 reference mAbs or 63 plasma samples was measured, generating a neutralization profile (i.e., ranking of relative neutralization of each HCVpp) for each reference mAb ("mAb rank") and each plasma sample ("plasma rank"). Points on each graph represent the ranking of relative neutralization sensitivity of each of 19 HCVpp by plasma on the $x$ axis and a reference mAb on the $y$ axis. Reference $\mathrm{mAb}$ neutralization profiles were added in all possible proportions to generate an array of possible combined mAb neutralization profiles ("combined mAb rank"). The algorithm then identified the specific combined reference mAb neutralization profile with the minimum difference from each plasma neutralization profile, thereby delineating the most likely proportion of each reference mAb contributing to the neutralization profile of the plasma sample.

in individuals with subsequent clearance or persistence of $\mathrm{HCV}$ can be attributed to a set of NAbs with known epitope specificities. Notably, focusing of the immune response toward bNAbs rather than narrow-breadth NAbs and simultaneous expression of multiple bNAb types was associated with greater plasma neutralizing breadth and with HCV clearance. These studies inform new strategies for vaccine development by identifying bNAb combinations in plasma associated with $\mathrm{HCV}$ clearance, while also providing a high-throughput assay that could identify these responses after vaccination trials.

\section{Results}

Deconvolution method. We previously measured neutralization of a diverse panel of 19 genotype $1 \mathrm{a}$ and $1 \mathrm{~b} \mathrm{HCV}$ pseudoparticles (HCVpp) by 20 anti-HCVhuman mAbs $(13,28)$. The HCVpp panel was selected to maximize genetic diversity among functional genotype $1 \mathrm{HCV}$ E1/E2 genes, and it contains $94 \%$ of amino acid polymorphisms present at greater than $5 \%$ frequency in genotype $1 \mathrm{HCV}$ isolates from GenBank (https://www.ncbi.nlm. nih.gov/genbank/) $(29,30)$. We have previously observed concordance between the neutralization breadth of mAbs measured using this diverse genotype 1 panel and neutralizing breadth of the same mAbs measured using HCV strains from genotypes 1-6 (13, 16, 30). Each mAb exhibited a distinct ranking of relative neutralization potency across the HCVpp in the panel, also known as a neutralization fingerprint or neutralization profile. The neutralization profile of each $\mathrm{mAb}$ was unique and reproducible, and these neutralization profiles also could be used to cluster functionally similar mAbs into groups (28). Subsequently, we observed that plasma samples from persons infected with $\mathrm{HCV}$ also exhibited distinct neutralization profiles across the HCVpp panel (18), leading us to hypothesize that these neu- tralization profiles could be used to deconvolute HCV-specific NAbs in polyclonal plasma.

To deconvolute HCV-specific NAbs in plasma, we measured neutralization of the 19-HCVpp panel by plasma samples at a 1:100 or 1:20 dilution, generating a neutralization profile for each sample (Figure 1). To analyze these data, we adapted an algorithm developed by Georgiev et al. for deconvolution of HIV-specific NAbs (31). This algorithm adds reference $\mathrm{mAb}$ neutralization profiles in all possible proportions to generate an array of combined reference $\mathrm{mAb}$ neutralization profiles. The algorithm then identifies the combined reference $\mathrm{mAb}$ neutralization profile with the minimum difference from the plasma neutralization profile, thereby delineating the most likely proportion of each reference mAb contributing to the neutralization profile of the plasma sample.

We added an additional step to the algorithm to quantitate quality of fit of each plasma deconvolution (see Methods and Supplemental Figure 1; supplemental material available online with this article; https://doi.org/10.1172/JCI130720DS1). For this quality analysis, we added reference $\mathrm{mAb}$ neutralization profiles in the proportions determined by the deconvolution and then compared the resulting combined $\mathrm{mAb}$ neutralization profile with the corresponding plasma neutralization profile using Pearson's correlation. A deconvolution was considered a good fit if the $P$ value of this correlation was less than 0.05 .

Selection of a mAb reference panel for plasma deconvolution. Many mAbs targeting distinct epitopes on HCV E1/E2 have been isolated from the B cells of HCV-infected humans, but it is not known which of these mAbs might contribute to plasma neutralizing activity. To identify the most comprehensive, least redundant $\mathrm{mAb}$ reference panel for plasma deconvolution analysis, we analyzed our previously tested set of $20 \mathrm{mAbs}$. This set includes mAbs specific for a variety of epitopes on E1 and/or E2 (Figure 2A), and it includes 
A

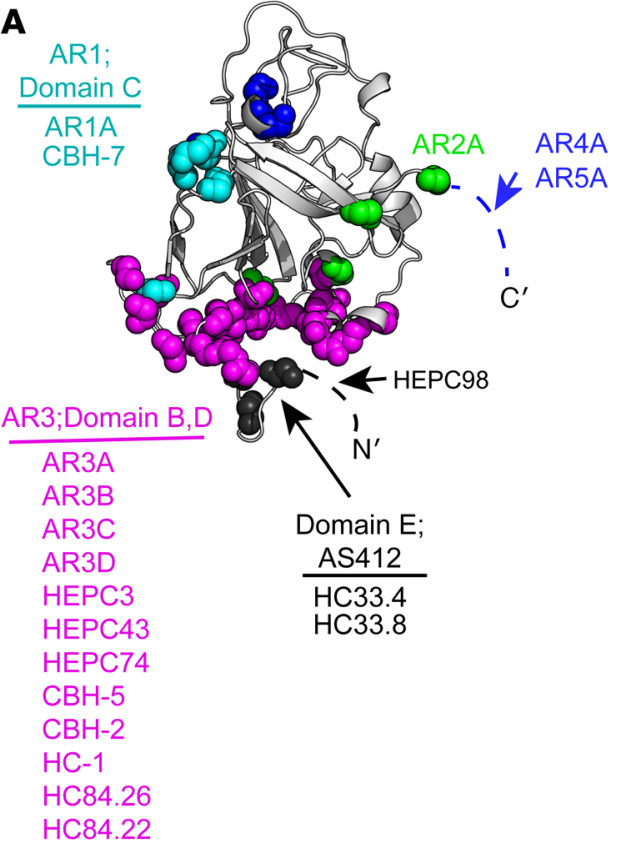

B

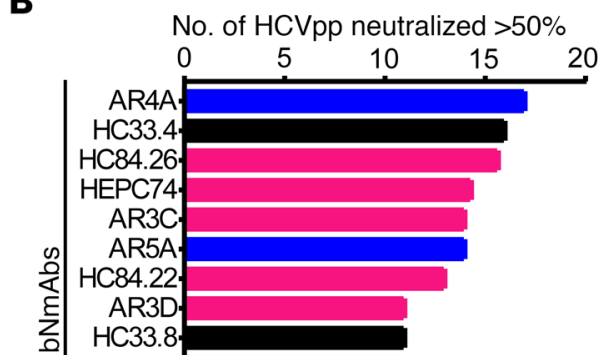

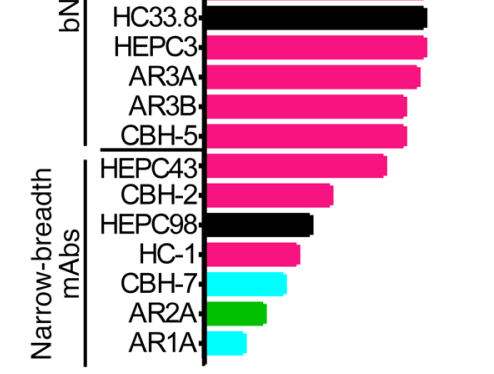

Figure 2. Selection of a reference mAb panel. (A) Main-chain atoms of previously defined mAb binding residues identified by alanine-scanning mutagenesis (see Methods) marked with colored spheres on the crystallized structure of the HCV E2 protein ectodomain (gray), strain 1609, PDB accession 6MEI (15), with colors modified in PyMOL, v1.8.6.2. For clarity, a maximum of 5 residues with impact on binding of each mAb are marked. Antigenic domains/antigenic regions (ARs) are indicated, and mAbs highlighted with the same color share multiple binding residues. Dashed lines indicate a disordered region (HVR1) or part of the E2 protein truncated to aid crystallization (stem region of E2). Putative AR4A and AR5A binding residues on $\mathrm{E} 1$ are not shown. (B) Neutralizing breadth of reference mAbs at $10 \mu \mathrm{g} / \mathrm{mL}$ measured with a panel of 19 genotype $1 \mathrm{HCVpp}$, with a threshold of $50 \%$ neutralization considered positive. (C) Functional grouping of reference $m A b s$. For each $m A b$, neutralization of each of 19 HCVpp was measured in duplicate, generating a neutralization profile (i.e., ranking of relative neutralization of each HCVpp) (Supplemental Figure 2), and pairwise Pearson correlations were measured between these neutralization profiles. Circles at each intersection of the heatmap are scaled by the magnitude of the correlation $(r)$ between the indicated NAbs. mAbs were grouped into an NAb type if the neutralization profile of each $\mathrm{mAb}$ in the group had a correlation of greater than or equal to 0.55 with every other mAb in the group. One representative $m A b$ (indicated with an asterisk) was selected from each NAb type to form the final panel of 8 reference mAbs. some of the most broadly neutralizing mAbs described to date, as well as mAbs with more limited neutralizing breadth (Figure $2 \mathrm{~B}$ and refs. 13, 19, 20, 26, 32, 33). To cluster this set of mAbs into NAb types based on functional similarity, we calculated pairwise Pearson correlations between $\mathrm{mAb}$ neutralization profiles, and mAbs were grouped if the neutralization profile of each $\mathrm{mAb}$ in the group had a correlation $(r)$ greater than or equal to $0.55(P<0.02)$ with the profile of every other mAb in the group (Figure 2C and Supplemental Figure 2). At this $r$-value threshold, most mAbs with highly similar binding sites fell into the same group, and most mAbs with clearly distinct binding sites fell into distinct groups. For example, mAbs AR3A, AR3B, AR3C, and AR3D, which bind overlapping epitopes on E2 and compete for E2 binding $(19,34,35)$, formed NAb type 1. HC33.4, HC33.8, and HEPC98, which each bind near the $\mathrm{N}$-terminus of E2, and also compete for E2 binding $(13,26,27)$, formed NAb type 2 . As we have previously noted, this analysis also revealed some functional relationships among mAbs that were not predicted by their binding sites (28). Some mAbs, which are thought to bind to distinct antigenic sites, like $\mathrm{HC}-1$ and $\mathrm{CBH}-7$, appeared to be functionally related, forming a single NAb type (Nab type 4). Conversely, some mAbs binding to partially overlapping epitopes, like AR3A and HC-1 $(36,37)$, segregated into distinct NAb types (NAb type 1 and NAb type 4, respectively). Some of these unexpected functional relationships are due to shared resistance mutations outside of the defined epitopes, which likely mediate indirect effects by allosteric means $(28,38-40)$. Overall, these data confirm that neutralization profiles can be used to identify individual mAbs or groups of mAbs with closely related binding epitopes.

Based on correlations between neutralization profiles, 18 mAbs segregated into 6 NAb types including 2 to 4 mAbs each, 
Reference mAbs

\begin{tabular}{|c|c|c|c|c|c|c|c|c|c|}
\hline mAbs added & $\underset{\frac{\alpha}{\alpha}}{\frac{\zeta}{\alpha}}$ & ํㅗ & $\begin{array}{l}\infty \\
\stackrel{\circ}{0} \\
0 \\
\text { Ш }\end{array}$ & $\begin{array}{l}\frac{N}{1} \\
\frac{1}{0} \\
0\end{array}$ & 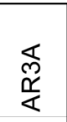 & 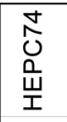 & 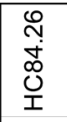 & $\underset{\substack{\alpha \\
\ll}}{\overleftarrow{\alpha}}$ & 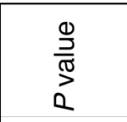 \\
\hline AR1A & 0.99 & 0.00 & 0.01 & 0.00 & 0.00 & 0.00 & 0.00 & 0.00 & $<1 \times 10^{-6}$ \\
\hline $\mathrm{HC}-1$ & 0.00 & 0.94 & 0.00 & 0.00 & 0.03 & 0.00 & 0.03 & 0.00 & $<1 \times 10^{-6}$ \\
\hline HEPC 98 & 0.07 & 0.01 & 0.90 & 0.00 & 0.00 & 0.00 & 0.00 & 0.02 & $<1 \times 10^{-6}$ \\
\hline $\mathrm{CBH}-2$ & 0.00 & 0.00 & 0.00 & 1.00 & 0.00 & 0.00 & 0.00 & 0.00 & $<1 \times 10^{-6}$ \\
\hline AR3A & 0.00 & 0.03 & 0.00 & 0.00 & 0.97 & 0.00 & 0.00 & 0.00 & $<1 \times 10^{-6}$ \\
\hline HEPC74 & 0.00 & 0.00 & 0.02 & 0.00 & 0.00 & 0.98 & 0.00 & 0.00 & $<1 \times 10^{-6}$ \\
\hline $\mathrm{HC} 84.26$ & 0.02 & 0.00 & 0.00 & 0.00 & 0.00 & 0.00 & 0.97 & 0.01 & $<1 \times 10^{-6}$ \\
\hline AR4A & 0.00 & 0.01 & 0.00 & 0.00 & 0.00 & 0.00 & 0.07 & 0.92 & $<1 \times 10^{-6}$ \\
\hline $\mathrm{HC}-1+\mathrm{AR} 3 \mathrm{~A}$ & 0.00 & 0.29 & 0.00 & 0.00 & 0.71 & 0.00 & 0.00 & 0.00 & $<1 \times 10^{-6}$ \\
\hline $\mathrm{CBH}-2+\mathrm{AR} 3 \mathrm{~A}$ & 0.00 & 0.00 & 0.00 & 0.11 & 0.89 & 0.00 & 0.00 & 0.00 & $<1 \times 10^{-6}$ \\
\hline$A R 1 A+A R 4 A$ & 0.00 & 0.03 & 0.00 & 0.00 & 0.00 & 0.07 & 0.08 & 0.82 & $<1 \times 10^{-6}$ \\
\hline $\mathrm{HC}-1+\mathrm{CBH}-2+\mathrm{AR} 3 \mathrm{~A}$ & 0.00 & 0.26 & 0.00 & 0.22 & 0.53 & 0.00 & 0.00 & 0.00 & $5 \times 10^{-5}$ \\
\hline HEPC98 + HEPC74 + HC84.26 & 0.00 & 0.07 & 0.26 & 0.00 & 0.00 & 0.33 & 0.34 & 0.00 & $<1 \times 10^{-6}$ \\
\hline
\end{tabular}

Figure 3. Deconvolution of samples containing known mAbs. Neutralization profiles of samples containing single-reference $m A b s$ or combinations of reference mAbs were used as input into the deconvolution algorithm. Each 2- or $3-\mathrm{mAb}$ combination included $5 \mu \mathrm{g} / \mathrm{mL}$ or $3.3 \mu \mathrm{g} / \mathrm{mL}$ of each $m A b$, respectively. These neutralization profiles were determined in a single experiment, with neutralization of each HCVpp measured in duplicate. Reference mAb profiles were averaged from 4 or 5 independent experiments. Values are the proportion of the neutralization activity of the sample attributed to each reference mAb by the deconvolution analysis. Proportions greater than 0.10 are highlighted in gray. $P$ values indicate the Pearson correlation of the neutralization profiles of spiked-in mAbs with a combined reference $\mathrm{mAb}$ neutralization profile comprising the indicated proportion of each reference mAb (see Methods and Supplemental Figure 1). and $2 \mathrm{mAbs}, \mathrm{CBH}-2$ and AR1A, did not group with other mAbs (Figure 2C). To select the $\mathrm{mAb}$ reference panel for plasma deconvolution analysis, we identified the most functionally distinct representative of each NAb type. This was the mAb from each group with the neutralization profile that had the lowest correlations with the neutralization profiles of mAbs from other NAb types. This analysis selected a reference panel comprising the following 8 mAbs: AR3A, HEPC98, HEPC74, HC-1, AR4A, HC84.26, CBH-2, and AR1A. Notably, reference mAbs in this set exhibited a range of neutralizing breadth, from 3 of 19 HCVpp neutralized by $10 \mu \mathrm{g} / \mathrm{mL}$ of AR1A to 17 of $19 \mathrm{HCVpp}$ neutralized by $10 \mu \mathrm{g} / \mathrm{mL}$ of AR4A. For the purposes of this study, we defined bNAbs as NAbs capable of neutralizing greater than or equal to 10 of 19 HCVpp by greater than $50 \%$ when tested at $10 \mu \mathrm{g} / \mathrm{mL}$ concentrations and narrow-breadth NAbs as NAbs neutralizing less than 10 of 19 HCVpp under these same conditions. By these criteria, 4 of 8 reference mAbs were bNAbs (AR4A, HC84.26, HEPC74, and AR3A) and 4 of 8 were narrow-breadth NAbs (AR1A, HC-1, HEPC98, and CBH-2).

Deconvolution of samples containing known mAbs. To validate the $\mathrm{mAb}$ reference panel and deconvolution method, we repeated neutralization testing against the $19-\mathrm{HCVpp}$ panel with singlereference $\mathrm{mAbs}$ or combinations of reference mAbs. We then used these neutralization profiles as input into the deconvolution algorithm (Figure 3). As expected, deconvolution analysis correctly identified the $\mathrm{mAb}$ present in each single-mAb sample. Notably, these experiments confirmed that the deconvolution method can reliably discriminate between each of the 8 reference mAbs, including AR3A, HEPC74, HC84.26, CBH-2, and $\mathrm{HC}-1$, which target overlapping epitopes in the E2 front layer. We also analyzed samples containing combinations of 2 or 3 reference mAbs, with each reference $\mathrm{mAb}$ included in at least 1 combination. All $\mathrm{mAbs}$ except AR1A were identified correctly in the 2-mAb combinations of HC-1+AR3A, CBH-2+AR3A, and AR1A+AR4A. Notably, proportions assigned to each $\mathrm{mAb}$ in the $2-\mathrm{mAb}$ combinations were not equivalent, although the $\mathrm{mAbs}$ were combined at equivalent concentrations, likely reflecting relative potency of the mAbs.
We also tested 2 different combinations of 3 mAbs. When HC-1, $\mathrm{CBH}-2$, and AR3A were combined at equivalent concentrations, all 3 mAbs were identified in the deconvolution analysis at proportions of 0.26, 0.22, and 0.53, respectively. HEPC98, HEPC74, and HC84.26 also were identified correctly in combination, with proportions of $0.26,0.33$, and 0.34 , respectively. Taken together, these data confirm that deconvolution analysis can identify individual $\mathrm{mAbs}$ alone or in polyclonal mixtures, although some mAbs, such as AR1A, may be more difficult to detect in a mixture with other mAbs.

Because low-level false-positive proportions were identified in some samples, we set a true-positive threshold proportion greater than 0.1 , which resulted in $95 \%$ sensitivity and $100 \%$ specificity in Figure 3 experiments. Proportions greater than 0.1 were considered positive in all subsequent analyses.

Because neutralization profiles are a relative ranking of neutralization of HCVpp by each NAb, rather than absolute neutralization values, we hypothesized that deconvolution results would be consistent across multiple mAb concentrations. To test this hypothesis, we performed deconvolution analysis using neutralization profile results measured using 2 different concentrations of each reference $\mathrm{mAb}$ (Supplemental Figure 3). The deconvolution result for each $\mathrm{mAb}$ was consistent at either 10 or $2 \mu \mathrm{g} / \mathrm{mL}$ mAb concentrations (50 or $10 \mu \mathrm{g} / \mathrm{mL}$ for AR1A), confirming that neutralization profiles remain consistent even when percentage neutralization values are very low. Notably, narrow-breadth mAbs AR1A, HC-1, and $\mathrm{CBH}-2$ were correctly identified based on relative neutralization of HCVpp across the panel even at concentrations of 2 to 10 $\mu \mathrm{g} / \mathrm{mL}$, when each $\mathrm{mAb}$ neutralized only 0 or 1 of $19 \mathrm{HCVpp}$ in the panel by more than $50 \%$, and 3 to $6 \mathrm{HCVpp}$ by more than $25 \%$.

Concordance between plasma NAb deconvolution and mAbs isolated from $B$ cells of the same subject. To validate the plasma deconvolution method by a second approach, we performed deconvolution analysis on plasma of 2 human donors, subjects C117 and C110, from whom we had previously isolated mAbs from peripheral blood B cells (Figure 4). C117 was the donor of B cells producing reference mAb HEPC98 as well as mAbs designated HEPC3 and 

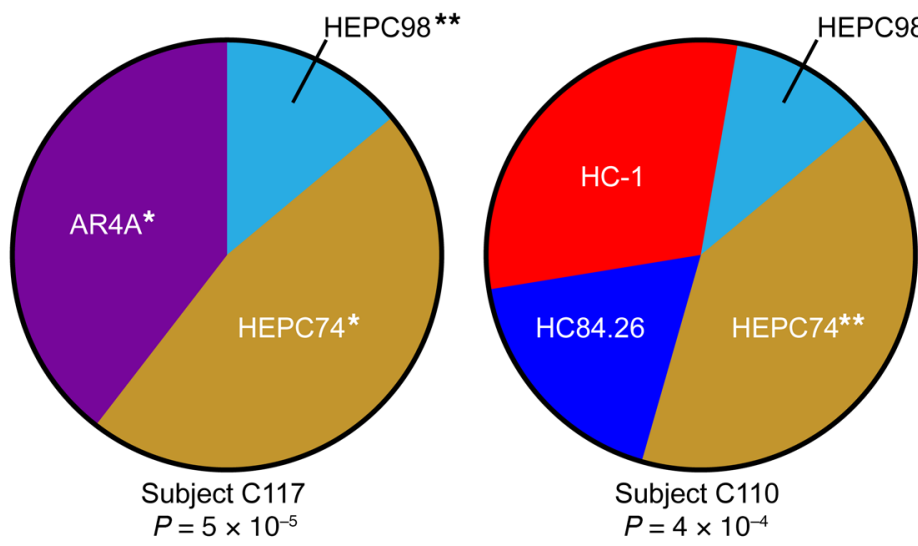

Figure 4. Concordance between plasma deconvolution and mAbs isolated from B cells of the same subject. Deconvolution of NAb types in plasma of 2 subjects, C117 and C110, from whom reference mAbs were also isolated from B cells. Plasma neutralization profiles were averaged from 2 independent experiments, which were each performed in duplicate. Reference mAb profiles were averaged from 5 independent experiments, with each performed in duplicate. Wedge sizes are proportional to the plasma response attributed to each reference $\mathrm{mAb} .{ }^{* *}$ Reference $\mathrm{mAb}$ detected in plasma was isolated from the $B$ cells of the same subject. ${ }^{*}$ Reference $\mathrm{mAb}$ detected in plasma and a mAb isolated from the $B$ cells of this subject are of the same NAb type (i.e., they have positively correlated neutralization profiles and compete for E1/E2 binding). $P$ values are for Pearson correlations of each plasma neutralization profile with a combined reference $\mathrm{mAb}$ neutralization profile comprising the indicated proportion of each reference $\mathrm{mAb}$.
HEPC84, which are HEPC74-type or AR4A-type NAbs, respectively (Figure 2 and ref. 13). Therefore, we hypothesized that we should detect HEPC98-type, HEPC74-type, and AR4A-type NAbs in $\mathrm{C} 117$ plasma. C110 was the donor of B cells producing reference mAb HEPC74, so we hypothesized that we should detect HEPC74-type NAbs in C110 plasma. For each subject, we analyzed plasma obtained prior to HCV clearance, using the averaged neutralization profiles obtained using 1:20 and 1:100 dilutions of each plasma sample as input into the deconvolution analysis (see Methods). As expected, HEPC98-type, HEPC74-type, and AR4A-type NAbs were each identified in C117 plasma (proportions $=0.12$, 0.40, and 0.34, respectively), and HEPC74-type NAbs were identified in C110 plasma (proportion $=0.36$ ). Notably, HC-1-type, HC84.26-type, and HEPC98-type NAbs were also detected in C110 plasma (proportions $=0.27,0.16$, and 0.10 , respectively), although they were not isolated from the subject's B cells, which may reflect false-positive plasma deconvolution results or the relative inefficiency of $\mathrm{mAb}$ isolation. Taken together, these results show that $\mathrm{mAbs}$ isolated from the B cells of 2 subjects also were identified in the plasma of the same subjects by deconvolution analysis.

To further validate the deconvolution method, we prospectively deconvoluted the plasma NAbs of a different HCV-positive subject, designated $\mathrm{C} 18$, using an average of neutralization profiles obtained using 1:20 and 1:100 dilutions of plasma as input into the deconvolution analysis. We then isolated $\mathrm{mAbs}$ from $\mathrm{B}$ cells of the same subject (Figure 5). Unlike C117 and C110, some of the neutralizing activity in C18 plasma was attributed to AR1A-type and AR3A-type NAbs (proportions 0.16 and 0.13 , respectively), with the remainder of activity attributed to HEPC98-type and AR4A-type NAbs (proportions 0.37 and 0.33 , respectively) (Figure $5 \mathrm{~A}$ ). We recently described isolation and characterization of $13 \mathrm{mAbs}$ from $\mathrm{B}$ cells of this subject (16). In that study, 12 of 13 mAbs displayed neutralizing activity across the HCVpp panel at a $50 \mu \mathrm{g} / \mathrm{mL}$ concentration. We measured neutralization profiles for these $12 \mathrm{mAbs}$, and we also mapped up to 15 (range, 3 to 15) probable E1/E2-binding residues for each C18 mAb and reference $\mathrm{mAb}$ based on binding to a comprehensive alanine mutant library spanning the E1 and E2 proteins of strain H77. Here, we identified the reference mAb (AR1A, HC-1, CBH-2, HEPC98, AR3A, HEPC74, HC84.26, or AR4A) that was most similar to each $\mathrm{C} 18 \mathrm{mAb}$ based on neutralization profile correlations and/or shared probable E1/E2 binding residues (Figure $5 \mathrm{~B}$ ).
Neutralization profiles from 10 of 12 mAbs isolated from C18 $\mathrm{B}$ cells correlated significantly with profiles of reference $\mathrm{mAb}$ types identified in plasma by the deconvolution analysis (AR1A, AR3A, or AR4A), which was significantly greater than the concordance between C18 plasma NAbs and C18 B cell mAbs expected by chance ( $83 \%$ vs. $44 \%, P=0.007$ by binomial test, see Methods). Nine of 12 C18 mAbs (75\%) shared both neutralization profiles and E1/E2 binding residues with reference mAb types identified in plasma by the deconvolution analysis. In addition, E1/E2 binding residues of $\mathrm{C} 18 \mathrm{~B}$ cell $\mathrm{mAbs}$ were 7.5-fold more likely to fall at binding residues of reference $\mathrm{mAb}$ types identified in plasma by deconvolution analysis than at other residues in E1 or E2 $(P<1 \times$ $10^{-15}$ by Fisher's exact test).

Three of 12 C18 B cell mAbs (25\%) were not predicted by the plasma deconvolution analysis. Two $\mathrm{C} 18 \mathrm{mAbs}$ matched best with reference $\mathrm{mAb} \mathrm{HEPC74}$, which was not detected in plasma. In addition, C18 mAb HEPC112 did not share E1/E2 binding residues with any reference $\mathrm{mAb}$, although the HEPC112 neutralization profile correlated strongly with reference mAb AR1A. This correlation between neutralization profiles of AR1A and HEPC112 is explained in part by the strong bias of each $\mathrm{mAb}$ for neutralization of genotype 1a strains over $1 \mathrm{~b}$ strains, indicating that this method may falsely attribute some genotype 1a-biased plasma NAb responses to AR1A-type NAbs. HEPC98-type NAbs were identified in plasma but not isolated from B cells, which may indicate a false-positive plasma deconvolution result or the relative inefficiency of $\mathrm{mAb}$ isolation.

Overall, 9 of 12 C18 mAbs isolated from B cells (75\%) matched plasma deconvolution-identified reference $\mathrm{mAbs}$ by both neutralization profiles and binding residues. Figure $5 \mathrm{C}$ shows the location on the E2 structure of probable binding residues of 3 reference $\mathrm{mAb}$ types identified in $\mathrm{C} 18$ plasma by the deconvolution analysis (AR1A, AR3A, AR4A) and 3 C18 mAbs with which each had a positively correlated neutralization profile and shared binding residues (HEPC151-2, HEPC154, HEPC130). Taken together, these data indicate that the NAb types detected in plasma by the deconvolution analysis were strongly predictive of both the functional phenotypes and $\mathrm{E} 1 / \mathrm{E} 2$ binding residues of mAbs subsequently isolated from $B$ cells of the same subject.

Deconvolution of NAb types in plasma of humans with subsequent clearance or persistence of $\mathrm{HCV}$ infection. For a prior study, we used 
A

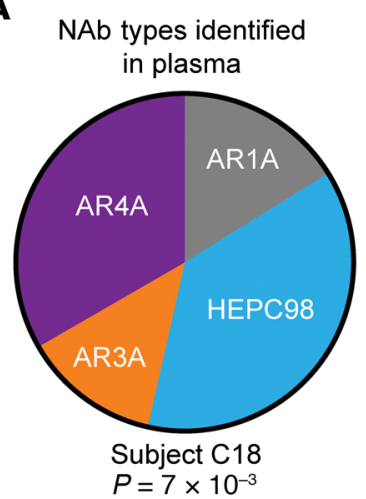

B

\begin{tabular}{ccccc} 
& & \multicolumn{2}{c}{$\begin{array}{c}\text { Neutralization } \\
\text { profile correlation }\end{array}$} & $\begin{array}{c}\text { Binding } \\
\text { residues } \\
\text { shared }\end{array}$ \\
\cline { 3 - 4 } C18 mAb & $\begin{array}{c}\text { Best match } \\
\text { reference mAb }\end{array}$ & $r$ & $P$ value & \\
HEPC151-2 & AR1A & 0.90 & $3 \times 10^{-7}$ & 7 \\
HEPC158 & AR1A & 0.82 & $3 \times 10^{-5}$ & 7 \\
HEPC167 & AR1A & 0.59 & $1 \times 10^{-2}$ & 7 \\
HEPC112 & AR1A & 0.80 & $7 \times 10^{-5}$ & 0 \\
HEPC154 & AR3A & 0.73 & $6 \times 10^{-4}$ & 7 \\
HEPC151-1 & AR3A & 0.69 & $1 \times 10^{-3}$ & 8 \\
HEPC146 & AR3A & 0.54 & $2 \times 10^{-2}$ & 2 \\
HEPC130 & AR4A & 0.60 & $8 \times 10^{-3}$ & 13 \\
HEPC111 & AR4A & 0.57 & $1 \times 10^{-2}$ & 12 \\
HEPC108 & AR4A & 0.60 & $9 \times 10^{-3}$ & 7 \\
HEPC153 & HEPC74 & 0.50 & $4 \times 10^{-2}$ & 10 \\
HEPC122 & HEPC74 & 0.42 & $9 \times 10^{-2}$ & 9 \\
\hline & & & &
\end{tabular}

C

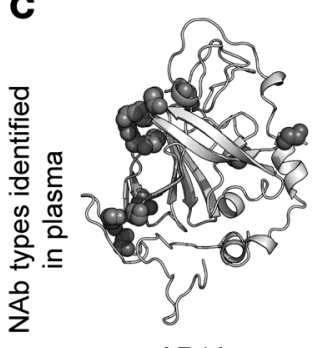

AR1A

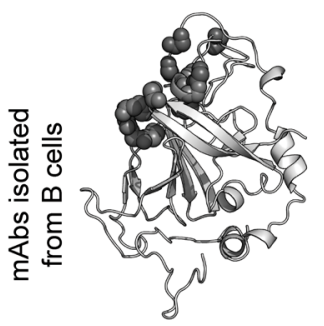

HEPC151-2

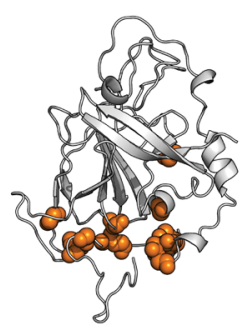

AR3A

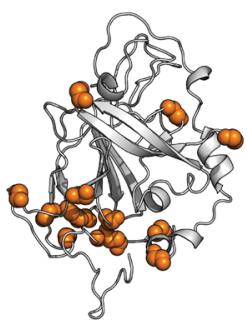

HEPC154

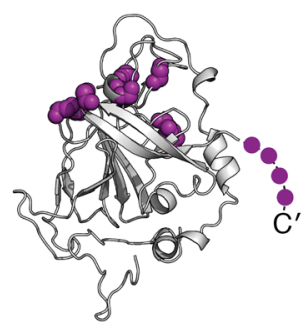

AR4A

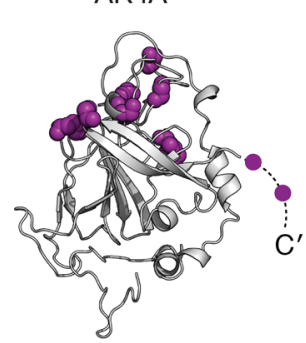

HEPC130
Figure 5. Plasma NAb deconvolution predicts mAbs subsequently isolated from $B$ cells of the same subject. (A) NAb deconvolution of subject C18 plasma. Plasma neutralization profile was averaged from 2 independent experiments. Reference $\mathrm{mAb}$ profiles were averaged from 5 independent experiments. Wedge sizes are proportional to the plasma response attributed to each reference $\mathrm{mAb} . P$ value is from the Pearson correlation of the C18 plasma neutralization profile with a combined neutralization profile comprising the indicated proportion of each reference mAb. (B) Pearson correlations between neutralization profiles and number of shared probable E1/E2-binding residues, as determined by alanine-scanning mutagenesis-E1/E2-binding analysis, between 12 mAbs isolated from subject C18 B cells and bestmatch reference mAbs. C18 mAb neutralization profiles were determined in a single experiment, with neutralization of each HCVpp measured in duplicate. (C) Examples of concordance between binding residues of reference $m A b s$ identified in plasma and 3 best-match mAbs isolated from C18 B cells. Colored spheres indicated main chain atoms of probable binding residues, superimposed on the crystallized structure of the HCV E2 protein ectodomain, strain 1b09, PDB accession 6MEI (15), with colors modified in PyMOL, v1.8.6.2. Shared putative E1 binding residues of HEPC130 and AR4A (Y201, N205) are not shown. the HCVpp panel to measure neutralizing breadth of plasma samples isolated from 21 subjects with subsequent clearance of HCV infection and 42 subjects with subsequent persistence of infection (11). Age, sex, race, follow-up interval, HCV infection genotype, and interferon- $\lambda$ 3-related rs12979860 allele frequencies were similar between these persistence and clearance groups, as previously described (11). Plasma was isolated from clearance subjects at the last viremic time point before infection clearance, and plasma from subjects in the persistence groups was time matched with the clearance plasma samples for the duration of infection. That study demonstrated that acute-infection plasma from clearance subjects was significantly more broadly neutralizing than plasma from subjects in the persistence group. We applied the deconvolution method to identify the NAbs responsible for this plasma neutralizing breadth.

Neutralization profiles of these 63 plasma samples were averaged from neutralization results measured in independent experiments at 1:20 and 1:100 plasma dilutions, and we used these neutralization profiles to deconvolute NAb types present in the samples (Figure 6). Forty-four (70\%) of the samples (persistence $n=29$, clearance $n=15$ ) had a good fit in the deconvolution anal- ysis $(P<0.05)$, suggesting that the neutralizing activity of these samples could be attributed to 1 or more of the mAb types in our reference panel. The proportion of subjects with good fit in the deconvolution analysis did not differ significantly between the persistence and clearance groups (69\% vs. $71 \%, P=1$, Fisher's exact test). Results for samples with poor fit in the deconvolution analysis are shown in Supplemental Figure 4. Although we could not identify the NAbs present in these samples with confidence, the samples with poor fit displayed very low neutralizing breadth (mean breadth $=1$ of 19 HCVpp neutralized), indicating that the poor fit could more likely be explained by absent or very low-titer bNAbs in these samples, rather than the presence of novel bNAbs not represented by the reference mAb panel.

We performed all subsequent analyses using only data from the plasma samples with good fit in the deconvolution analysis (Figures 7 and 8). A significantly higher proportion of the antibody response was devoted to narrow-breadth HC-1-type NAbs in persistence subjects relative to clearance subjects (mean persistence vs. clearance proportions 0.28 vs. $0.14, P=0.03$ by 1 -way ANOVA adjusted for multiple comparisons) (Figure 7A). The mean proportion of the antibody response devoted to each bNAb type (i.e., 


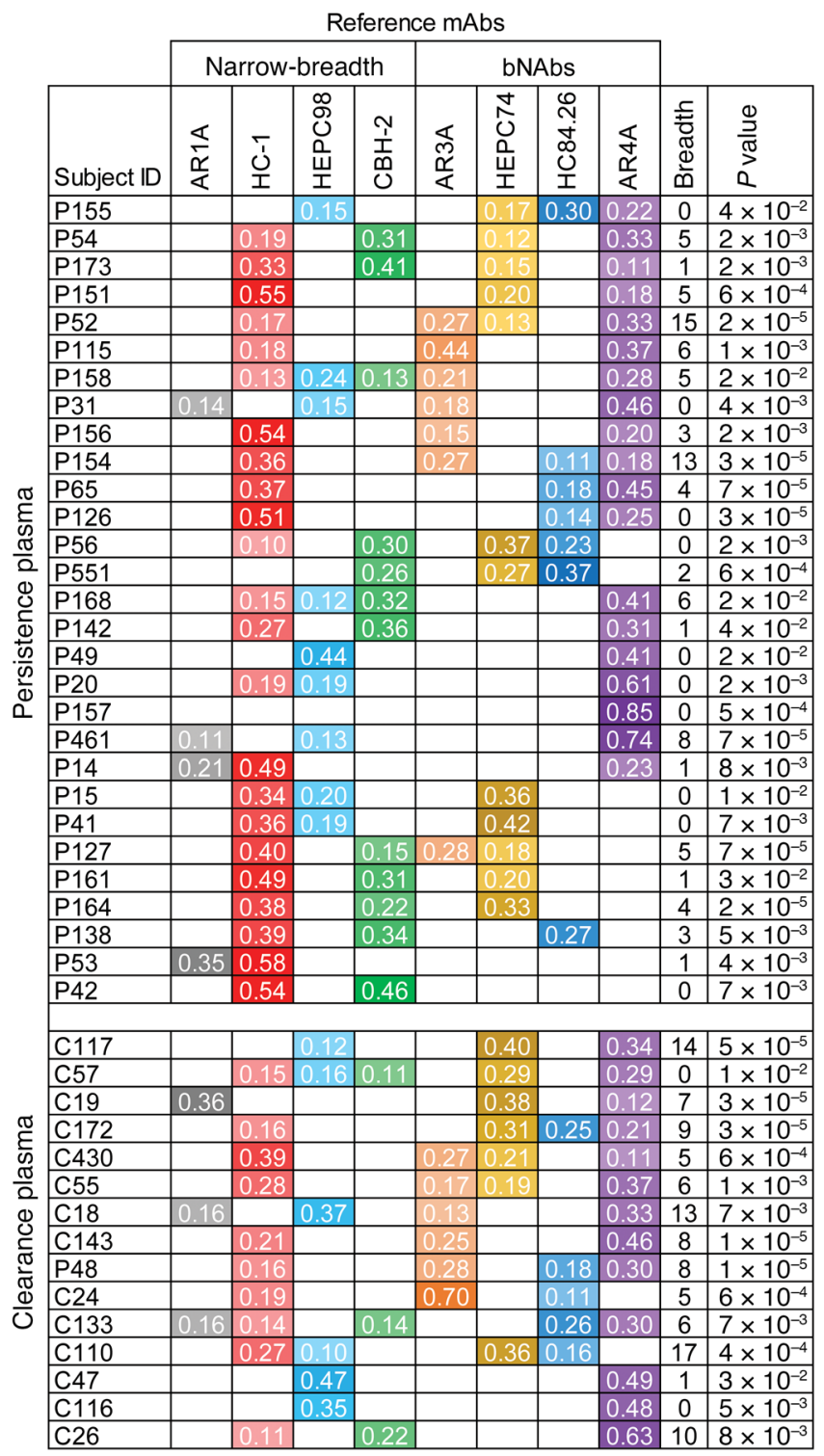

Figure 6. Deconvolution of NAbs in plasma of subjects with subsequent clearance or persistence of $\mathbf{H C V}$ infection. Reference mAbs are on the $x$ axis with plasma samples on the $y$ axis. Each plasma sample is from a different subject. Plasma neutralization profiles each were averaged from 2 independent experiments. Reference mAb profiles were averaged from 5 independent experiments. Values shown are the proportion of each plasma neutralizing response attributed to each reference mAb. Proportions greater than 0.1 are shown and marked with different colors for each NAb type, with higher values shaded darker. Plasma samples are grouped by subject outcome. Neutralizing breadth was calculated as the number out of 19 HCVpp neutralized greater than $50 \%$ by a 1:100 dilution of plasma. $P$ values are for the Pearson correlation between the plasma sample neutralization profile and the best-fit combined reference mAb neutralization profile. Only subjects with significant correlation $(P<0.05)$ between combined mAb neutralization profile and plasma neutralization profile are shown (persistence $n=29$, clearance $n=15$ ). Results for subjects with a poor deconvolution fit are shown in Supplemental Figure 4.

AR3A, HEPC74, HC84.26, or AR4A) was similar for persistence and clearance subjects, although there was a trend toward a higher proportion for each bNAb type in clearance relative to persistence subjects. Taken together, these data indicate that focusing of the immune response toward narrow-breadth HC-1-type NAbs was associated with the persistence of $\mathrm{HCV}$ infection, and no single NAb type was strongly associated with HCV clearance.

Given prior data from our group and others indicating that some bNAbs in combination are synergistic with enhanced neutralizing breadth $(16,41,42)$, we hypothesized that combinations of bNAbs, rather than individual responses, might be associated with HCV clearance. Therefore, we compared the total proportion of the neutralizing response devoted to bNAbs (i.e., sum of AR4A, HC84.26, HEPC74, and AR3A proportions) in clearance or persistence subjects (Figure 7B). Remarkably, we measured significantly higher total bNAb proportions in clearance vs. persistence subjects (mean proportion, 0.62 vs. $0.46 ; P=$ 0.009 by 2-tailed $t$ test). Although the total number of reference NAb types detected per plasma sample did not differ between clearance and persistence groups (mean $=3.5$ vs. 3.2 NAb types, $P=0.24$ by 2-tailed $t$ test) (Figure 7C), we detected a significantly higher number of distinct bNAb types per plasma sample in clearance vs. persistence subjects (mean no. of bNAb types per sample 2.1 vs. $1.6, P=0.04$ by 2-tailed $t$ test) (Figure 7D). The most common $2 \mathrm{bNAb}$ combinations detected in clearance plasma were HEPC74+AR4A (6 of 15 subjects), AR3A+AR4A (5 of 15 subjects), and HC84.26+AR4A (3 of 15 subjects) (Figure $7 \mathrm{E}$ ). These data indicate that focusing of the humoral response toward bNAbs rather than narrow-breadth NAbs and simultaneous expression of multiple bNAb types in a single plasma sample was associated with HCV clearance.

Focusing of the humoral response toward bNAbs and expression of multiple bNAb types was associated with greater plasma neutralizing breadth. To investigate the mechanism by which focusing of the humoral response toward bNAbs and expression of multiple bNAb-types was associated with infection clearance, we measured the association between these parameters and plasma neutralizing breadth. First, we compared neutralizing breadth of persistence and clearance plasma samples, limiting the analysis to samples with good fit in the deconvolution analysis (Figure 8A). We observed significantly greater neutralizing breadth by the clearance samples (mean no. of HCVpp neutralized by 1:100 dilution of clearance vs. persistence plasma 7.3 vs. $3.1, P=0.003$ by 2-tailed $t$ test), which was consistent with our prior analysis of the full set of 63 plasma samples (11).

Next, we measured the correlation between the total bNAb proportion of each plasma sample (i.e., sum of AR4A, HC84.26, HEPC74, and AR3A proportions) and the neutralizing breadth of that sample (Figure 8B). We observed a statistically significant positive correlation between these variables $(r=0.39, P=0.01$ by Pearson's method). Notably, we also observed a significant correlation between the number of bNAb types expressed in each plasma sample and the neutralizing breadth of that sample (Figure 8C). The mean neutralizing breadth of plasma samples expressing 1 or fewer bNAb types, 2 bNAb types, or 3 bNAb types was 2.1, 5.3, or $8 \mathrm{HCVpp}$ neutralized, respectively $(P=0.004$ for the trend by 1-way ANOVA). Taken together, these data indicate that focusing of the humoral response toward bNAbs rather than narrow-breadth NAbs and simultaneous expression of multiple bNAb types was associated with greater plasma neutralizing breadth as well as clearance of HCV infection. 
A
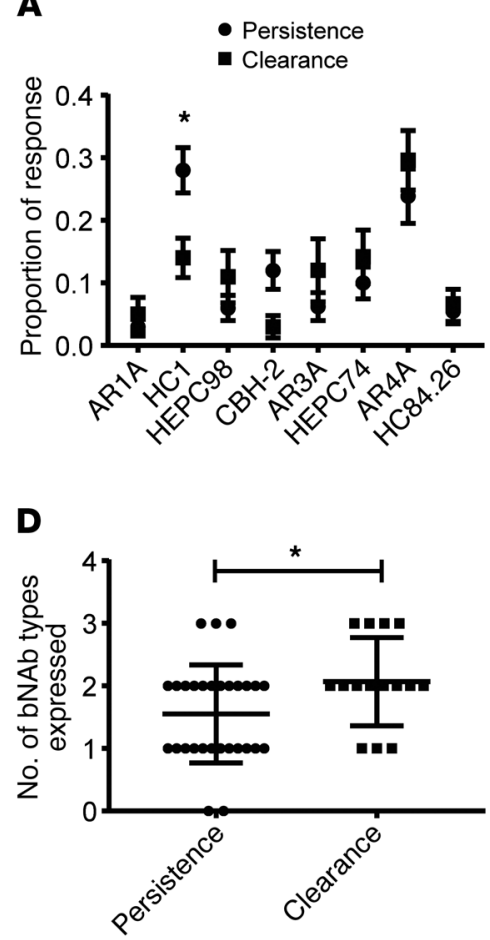

B

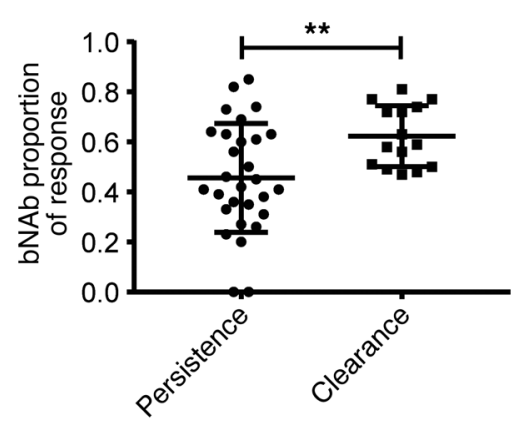

E

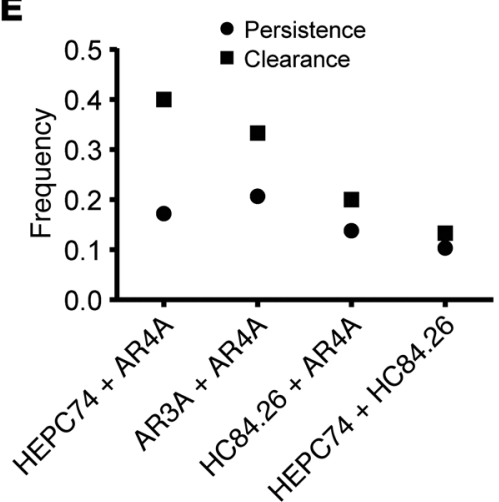

C

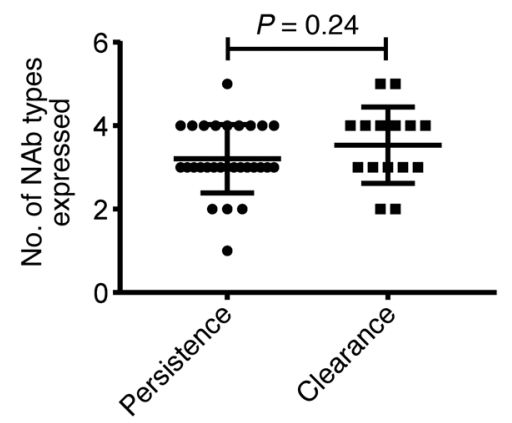

Figure 7. Focusing of the humoral response toward bNAbs and expression of multiple bNAb types was associated with HCV clearance. (A) Proportion of persistence and clearance plasma responses attributed to each NAb type. Values are means, and error bars are SEM. ${ }^{*} P<0.05$ by 1 -way ANOVA adjusted for multiple comparisons using Sidak's method. (B) Total proportion of persistence and clearance plasma responses attributed to bNAbs (AR3A, HEPC74, HC84.26, or AR4A). Horizontal lines are means, and whiskers are SD. ${ }^{*} P<0.01$ by 2-sided $t$ test. (C) Number of NAb types positive above a threshold of 0.10 for each plasma sample. Horizontal lines are means, and whiskers are SD. $P=0.24$ by 2 -sided $t$ test. (D) Number of bNAb types (AR3A, HEPC74, HC84.26, or AR4A) positive above a threshold of 0.10 for each plasma sample. Horizontal lines are means, and whiskers are SD. ${ }^{*} P<0.05$ by 2 -sided $t$ test. (E) Frequency of each observed bNAb combination across all persistence or clearance subjects.

\section{Discussion}

$\mathrm{HCV}$ vaccine development has been hampered by incomplete understanding of correlates of protective immunity in humans and by inadequate methods for assessing antibody responses induced by candidate vaccines. We have addressed these deficits through the development of an assay that deconvolutes the antiHCV NAbs in polyclonal plasma. Unexpectedly, we found that most human subjects with subsequent clearance or persistence of $\mathrm{HCV}$ infection developed at least $1 \mathrm{bNAb}$ during acute infection. Importantly, focusing of the humoral response toward bNAbs rather than narrow-breadth NAbs and simultaneous expression of multiple bNAb types was associated with greater plasma neutralizing breadth and with HCV clearance.

These results support prior work indicating that $\mathrm{HCV}$ vaccines should induce multiple bNAb types (19, 41-43), and we extend these findings by demonstrating that it may be important to induce HEPC74, AR3A, or HC84.26-like bNAbs in combination with AR4Alike bNAbs, while avoiding induction of more narrow-breadth NAbs like HC-1. Broadly neutralizing AR3A, HEPC74, and HC84.26 as well as relatively narrow-breadth $\mathrm{HC}-1$ and $\mathrm{CBH}-2$ bind to overlapping conformational epitopes, which may complicate efforts to induce $1 \mathrm{NAb}$ type without the other. It is possible that this complication has played a role in the relatively limited neutralizing breadth induced by candidate vaccines to date $(44,45)$, if HC-1-type NAbs were induced at the expense of more broadly neutralizing E2 front layer-specific antibodies. Targeting of more variable epitopes at the expense of more conserved, broadly neutralizing epitopes, commonly known as deceptive imprinting, has been described previously for HIV-1 and influenza (46). Alternatively, HC-1-type NAbs may represent an early response that could mature to a HEPC74 or AR3A-like response with appropriate $\mathrm{T}$ cell help. Further longitudinal studies of human subjects with persistent infection will be necessary to discriminate between these possibilities. Notably, traditional methods used to map epitopes of vaccine-induced NAbs, such as E1/E2 binding competition between serum and reference mAbs, would likely not discriminate between the desired E2 front layer-specific bNAbs and less desirable narrow-breadth HC-1-type NAbs targeting the same antigenic site. This deconvolution method also provides a considerable advantage relative to postvaccination neutralizing breadth testing alone because breadth might result from targeting of 1 or multiple epitopes. In contrast, deconvolution analysis identifies the epitopes targeted, which could facilitate rational antigen design and iterative vaccine improvement to favor induction of bNAb combinations associated with clearance of natural infection. Fortunately, this method is high throughput and requires only about $100 \mu \mathrm{L}$ of plasma, so it would be ideal for testing longitudinal plasma samples or for optimizing vaccine protocols when only small plasma volumes can be obtained. 
A

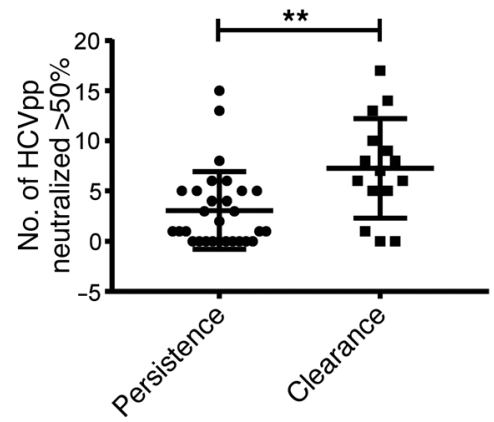

B

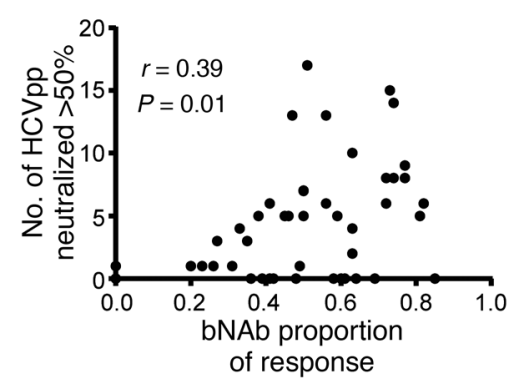

C

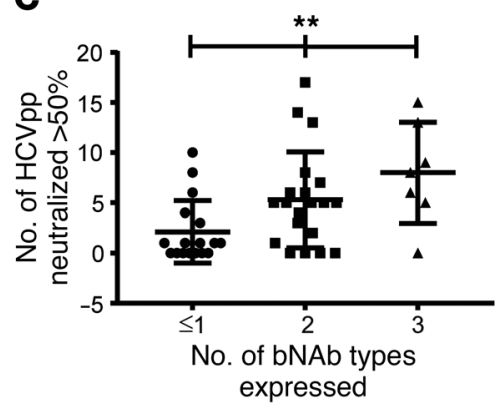

Figure 8. Focusing of the humoral response toward bNAbs and expression of multiple bNAb types was associated with greater plasma neutralizing breadth. (A) Neutralizing breadth of plasma samples from subjects in the persistence or clearance group (no. out of 19 HCVpp neutralized $>50 \%$ by a 1:100 dilution of plasma). (B) Correlation between total proportion of each plasma response attributed to bNAbs and neutralizing breadth of that plasma sample. $P$ and $r$ values were calculated by Pearson's method. (C) Relationship between the number of bNAb types expressed by each plasma sample and neutralizing breadth of that sample. ${ }^{* *} P<0.01$ for the trend by 1-way ANOVA.

This method has limitations. First, it is likely that epitopes and function of NAbs developing in different individuals do not exactly match the reference mAbs to which they are most closely related. These subtle differences may have important functional implications, so there is still a critical role for isolation of additional mAbs from B cells of HCV-infected or vaccinated subjects, which will allow molecular and structural characterization of protective or deleterious NAb-epitope interactions. The deconvolution assay likely can be improved iteratively by adding unique reference mAbs when they are identified and through the use of larger and more diverse HCVpp panels, which would likely improve discrimination between functionally related NAbs. Second, the assay appeared to favor detection of more potent NAbs over less potent NAbs, and therefore proportions of the neutralizing response attributed to individual mAbs in combination experiments did not always directly correlate with the relative concentrations of those mAbs in the mixture. Further standardization of the assay to quantitate actual concentrations of individual NAb types in plasma might therefore be difficult and would at minimum, require extensive testing of multiple-reference $\mathrm{mAb}$ combinations with each $\mathrm{mAb}$ at multiple different concentrations. The assay does not detect nonneutralizing antibodies, which may also play a role in controlling infection (47), or in shaping the NAb response by competing with NAbs for overlapping epitopes on E1 and E2 (22-27). The NAbs that were detected infrequently in this study may be rare in plasma, or they may be present but effectively out-competed for $\mathrm{E} 2$ binding by nonneutralizing antibodies. Further work is needed to understand this relationship between neutralizing and nonneutralizing antibodies against HCV. Finally, this method does not measure plasma neutralizing titers, which likely also play an important role in the outcome of infection.

In summary, we developed a method that deconvolutes antiHCV NAbs in polyclonal plasma, and we used this method to identify NAb types in acute plasma infection of human subjects with subsequent clearance or persistence of HCV. We found that no single Nab type was strongly associated with $\mathrm{HCV}$ clearance. Remarkably, however, focusing of the humoral response toward bNAbs rather than narrow-breadth NAbs and simultaneous expression of multiple bNAb types was associated with greater plasma neutralizing breadth and with HCV clearance. These studies inform new strategies for vaccine development by identifying bNAb combinations in plasma associated with clearance of HCV, while also providing a high-throughput assay that could identify these responses after vaccination trials.

\section{Methods}

Study subjects. Plasma samples and PBMCs were obtained from subjects in the Baltimore Before and After Acute Study of Hepatitis (BBAASH) cohort (10).

Source of mAbs. MAbs CBH-2, CBH-7, HC-1, CBH-5 (48), HC84.22, HC84.26 (20), HC33.4, and HC33.8 (26) were a gift from Steven Foung (Stanford University School of Medicine, Palo Alto, California, USA). mAbs AR1A, AR2A, AR3A, AR3B, AR3C, AR3D (19), AR4A, and AR5A (32) were a gift from Mansun Law (Scripps Research Institute, La Jolla, California, USA). All other antibodies were isolated in the laboratory of James E. Crowe Jr. $(13,16)$.

Cell lines. HEK293T cells and Hep3B cells were obtained from ATCC.

HCVpp neutralization assay. HCVpp were produced by lipofectamine-mediated transfection of HCV E1/E2, pNL4-3.Luc.R-E, and pAdVantage (Promega) plasmids into HEK293T cells as previously described $(28,49,50)$. Neutralization assays were performed as described previously $(51,52)$. mAbs at 10 or $50 \mu \mathrm{g} / \mathrm{mL}$, or heatinactivated plasma samples at 1:100 or 1:20 dilution were incubated with $\mathrm{HCVpp}$ for 1 hour at $37^{\circ} \mathrm{C}$ prior to addition to Hep3B cells in duplicate. Medium was changed after 5 hours, and cells were incubated for 72 hours before measuring luciferase activity in cell lysates in relative light units (RLUs). Only HCVpp preparations producing at least $1 \times 10^{6} \mathrm{RLU}$ were used for neutralization experiments, and HCVpp input was normalized to $1 \times 10^{6}$ to $6 \times 10^{6}$ RLUs. Nonspecific human IgG (SigmaAldrich) at $50 \mu \mathrm{g} / \mathrm{mL}$ was used as a negative control. Fraction unaffected $\left(\mathrm{F}_{\mathrm{u}}\right)$ was calculated by the formula: $\mathrm{F}_{\mathrm{u}}=\mathrm{RLUs}_{\mathrm{mAb}}$ or RLUs $\mathrm{s}_{\text {plasmal }} /$ RLUs $_{\text {nonspecific IgG }} F_{u}$ values were converted to a neutralization profile for each $\mathrm{mAb}$ or plasma sample (rank order of HCVpp neutralization with the most sensitive HCVpp ranked 1 and the least sensitive HCVpp ranked 19) for input into the deconvolution algorithm.

Deconvolution of NAb specificities in plasma. Neutralization of a diverse panel of 19 genotype 1 HCVpp (strains 1a09, 1a31, 1a38, 1a53, 
1a72, 1a80, 1a116, 1a123, 1a129, 1a142, 1a154, 1a157, 1b09, 1b14, 1b21, $1 \mathrm{~b} 34,1 \mathrm{~b} 38,1 \mathrm{~b} 52$, and $1 \mathrm{~b} 58$ ) by $20 \mathrm{HCV}$-specific mAbs at $10 \mu \mathrm{g} / \mathrm{mL}$ concentration was quantitated for prior studies, generating a neutralization profile for each $\mathrm{mAb}$, as previously described $(13,28)$. Once the final panel of 8 reference mAbs was selected, neutralization profiles were measured for each of these mAbs in 4 additional independent experiments. Neutralization profiles for each reference $\mathrm{mAb}$ were averaged across all 5 experiments by averaging the rank value for each HCVpp across all experiments, generating a final neutralization profile for each reference mAb (Supplemental Table 1). Plasma samples from subjects with clearance $(n=21)$ or persistence $(n=42)$ of HCV infection were previously tested at a 1:100 dilution for neutralization of the same 19 HCVpp panels (11), except that HCVpp 1b21 and 1a116 were replaced by closely related HCVpp 1 b20 (differing from 1 b21 by $1 \mathrm{aa}$ ) and 1a114 (differing from 1a116 by 10 aa), due to greater availability of these HCVpp stocks at the time of testing. Because reference mAbs were not tested against $1 \mathrm{~b} 20$ and 1a114, plasma neutralization results for these HCVpp were not included in neutralization profiles used for deconvolution analysis. The same plasma samples were tested in a second independent experiment at a 1:20 dilution for neutralization of the same 19-HCVpp panel used to determine reference $\mathrm{mAb}$ neutralization profiles, including strains $1 \mathrm{a} 116$ and $1 \mathrm{~b} 21$. Neutralization data for 8 HCVpp (1b34, 1a53, 1b38, 1a123, 1a80, 1b58, 1a142, and 1b14) were discarded from this second experiment due to inadequate HCVpp infectivity $\left(<1 \times 10^{6} \mathrm{RLU}\right)$. Neutralization profiles were averaged across these 2 independent experiments to generate a final neutralization profile for each plasma sample (Supplemental Table 2). Deconvolution analysis was performed using code developed by Georgiev et al. (31) (in Wolfram Mathematica, v. 11.0) to delineate the relative proportion of each reference $\mathrm{mAb}$ present in each plasma sample. Rank order of plasma neutralization profiles were reversed before analysis to fit input requirements of the deconvolution program.

Calculating fit of plasma deconvolution. (See example calculation, Supplemental Figure 1.) For each plasma sample deconvolution, a scaled neutralization profile was calculated for each reference $\mathrm{mAb}$ by multiplying the neutralization profile of that reference $\mathrm{mAb}$ by the proportion of that mAb type (range, $0-1$ ) calculated to be present in the plasma sample. These 8 scaled reference $\mathrm{mAb}$ neutralization profiles then were added to generate a combined reference $\mathrm{mAb}$ neutralization profile. This combined reference $\mathrm{mAb}$ neutralization profile was compared with the actual plasma neutralization profile by Pearson's correlation. NAb deconvolution for any plasma sample was considered a good fit if the correlation between the combined $\mathrm{mAb}$ neutralization profile and the plasma neutralization profile had a $P$ value less than 0.05 by 2 -sided test.

Shotgun mutagenesis epitope mapping. Epitope mapping of mAbs was previously described $(16,34)$. Briefly, comprehensive alaninescanning mutagenesis of an expression construct for HCV E1/E2 (genotype 1a, strain H77) changed each residue to alanine (with alanine residues changed to serine) to create a library of clones, each representing an individual point mutant, covering 552 of 555 target E1/E2 residues. Binding of each mAb to WT E1/E2 and to each of these alanine mutants was quantitated to identify up to 15 residues where mutation to alanine mediated the greatest reduction in mAb binding and also reduced binding by at least $50 \%$ relative to binding to WT H77 E1/E2. Probable binding residues of reference $\mathrm{mAbs}$ were identified through re-analysis of primary data included in prior publications $(13,36,37,48)$.

Statistics. Statistical analyses were performed in Prism (GraphPad Software, v7.02). Correlations between plasma and reference $\mathrm{mAb}$ neutralization profiles were calculated using Pearson's method. Proportion of persistence and clearance plasma responses attributed to each NAb type was compared by 1-way ANOVA adjusted for multiple comparisons using Sidak's method. The total proportion of persistence and clearance plasma responses attributed to bNAbs and numbers of $\mathrm{NAb}$ types per sample was compared by 2 -sided $t$ tests. The relationship between the number of bNAb types expressed and neutralizing breadth of samples was analyzed by 1-way ANOVA. For all comparisons, $P$ values less than 0.05 were considered significant. Probability of concordance between neutralization profiles of NAb types identified in C18 plasma and mAbs isolated from B cells arising by chance was calculated using a binomial test and the null hypothesis that each $\mathrm{mAb}$ isolated would fall by chance in 1 of 9 groups, either matching 1 of the 8 reference mAbs, or none of them. The significance of the increased likelihood of $\mathrm{C} 18 \mathrm{mAb}$ binding residues falling at binding residues of deconvolution-identified reference mAbs was calculated using Fisher's exact test and the following data: 8 to 15 binding residues were identified for each of $12 \mathrm{C} 18 \mathrm{mAbs} ; 3$ to 15 binding residues were known for each of $4 \mathrm{mAbs}$ identified in plasma; and there were 552 possible E1/E2 binding residues for each $\mathrm{mAb}$.

Study approval. The protocol was approved by the Institutional Review Board of the Johns Hopkins Hospital, and informed consent was obtained from all study participants.

\section{Author contributions}

JRB, SCR, and JEC conceived the study. VJK, MCM, MDC, and WOO performed experiments. JEC and AIF provided antibodies. VJK, GM, ALC, AIF, and JRB analyzed the data. VJK and JRB wrote the original draft. All authors reviewed and edited the manuscript.

\section{Acknowledgments}

The authors would like to thank Alexander Gooden for technical support. This research was supported by NIH grants R01 AI127469 (to JRB) and U19 AI088791 (to JRB and ALC). AIF is a Cancer Research Institute Irvington Fellow supported by the Cancer Research Institute. The content is solely the responsibility of the authors and does not necessarily represent the official views of the NIH.

Address correspondence to: Justin R. Bailey, 855 N. Wolfe Street, Rangos Building, Suite 520, Baltimore, Maryland 21205, USA. Phone: 410.614.6087; Email: jbailey7@jhmi.edu.
1. Gravitz L. Introduction: a smouldering public-health crisis. Nature. 2011;474(7350):S2-S4.

2. Holmberg SD, Spradling PR, Moorman AC, Denniston MM. Hepatitis C in the United States. N Engl J Med. 2013;368(20):1859-1861.

3. Falade-Nwulia O, Sulkowski M. The HCV care continuum does not end with cure: a call to arms for the prevention of reinfection. J Hepatol. 2017;66(2):267-269.

4. Midgard $\mathrm{H}$, et al. Hepatitis $\mathrm{C}$ reinfection after sustained virological response. J Hepatol. 2016;64(5):1020-1026.
5. Martin TC, et al. Hepatitis $\mathrm{C}$ virus reinfection incidence and treatment outcome among HIV-positive MSM. AIDS. 2013;27(16):2551-2557.

6. Pineda JA, et al. Hepatitis $\mathrm{C}$ virus reinfection after sustained virological response in HIV-infected patients with chronic hepatitis C. 
J Infect. 2015;71(5):571-577.

7. Martinello M, et al. $\mathrm{HCV}$ reinfection incidence among individuals treated for recent infection. JViral Hepat. 2017;24(5):359-370.

8. Mina MM, et al. Resistance to hepatitis $\mathrm{C}$ virus: potential genetic and immunological determinants. Lancet Infect Dis. 2015;15(4):451-460.

9. Bailey JR, Barnes E, Cox AL. Approaches, progress, and challenges to hepatitis $C$ vaccine development. Gastroenterology. 2019;156(2):418-430.

10. Cox AL, et al. Prospective evaluation of community-acquired acute-phase hepatitis $C$ virus infection. Clin Infect Dis. 2005;40(7):951-958.

11. Osburn WO, et al. Clearance of hepatitis $C$ infection is associated with the early appearance of broad neutralizing antibody responses. Hepatology. 2014;59(6):2140-2151.

12. Pestka JM, et al. Rapid induction of virusneutralizing antibodies and viral clearance in a single-source outbreak of hepatitis C. Proc Natl Acad Sci U S A. 2007;104(14):6025-6030.

13. Bailey JR, et al. Broadly neutralizing antibodies with few somatic mutations and hepatitis $\mathrm{C}$ virus clearance. JCI Insight. 2017;2(9):92872.

14. Merat $S J$, et al. Hepatitis $C$ virus broadly neutralizing monoclonal antibodies isolated 25 years after spontaneous clearance. PLoS One. 2016;11(10):e0165047.

15. Flyak AI, et al. HCV broadly neutralizing antibodies use a CDRH3 disulfide motif to recognize an E2 glycoprotein site that can be targeted for vaccine design. Cell Host Microbe. 2018;24(5):703-716.e3.

16. Colbert MD, et al. Broadly neutralizing antibodies targeting new sites of vulnerability in hepatitis C virus E1E2. J Virol. 2019;93(14):e02070-18.

17. Keck ZY, et al. Broadly neutralizing antibodies from an individual that naturally cleared multiple hepatitis $\mathrm{C}$ virus infections uncover molecular determinants for $\mathrm{E} 2$ targeting and vaccine design. PLoS Pathog. 2019;15(5):e1007772.

18. Kinchen VJ, et al. Broadly neutralizing antibody mediated clearance of human hepatitis C virus infection. Cell Host Microbe. 2018;24(5):717-730.e5.

19. Law M, et al. Broadly neutralizing antibodies protect against hepatitis $C$ virus quasispecies challenge. Nat Med.2008;14(1):25-27.

20. Keck ZY, et al. Human monoclonal antibodies to a novel cluster of conformational epitopes on HCV E2 with resistance to neutralization escape in a genotype 2a isolate. PLoS Pathog. 2012;8(4):e1002653.

21. Wong JA, et al. Recombinant hepatitis $\mathrm{C}$ virus envelope glycoprotein vaccine elicits antibodies targeting multiple epitopes on the envelope glycoproteins associated with broad cross-neutralization. JVirol. 2014;88(24):14278-14288.

22. Sabo MC, et al. Neutralizing monoclonal antibodies against hepatitis $\mathrm{C}$ virus $\mathrm{E} 2$ protein bind discontinuous epitopes and inhibit infection at a postattach- ment step. JVirol. 2011;85(14):7005-7019.

23. Zhang P, et al. Depletion of interfering antibodies in chronic hepatitis $C$ patients and vaccinated chimpanzees reveals broad cross-genotype neutralizing activity. Proc Natl Acad Sci U S A. 2009;106(18):7537-7541.

24. Kachko A, et al. Antibodies to an interfering epitope in hepatitis $C$ virus $E 2$ can mask vaccineinduced neutralizing activity. Hepatology. 2015;62(6):1670-1682.

25. Deng $L$, et al. Structural evidence for a bifurcated mode of action in the antibody-mediated neutralization of hepatitis C virus. Proc Natl Acad Sci U S A. 2013;110(18):7418-7422.

26. Keck Z, et al. Cooperativity in virus neutralization by human monoclonal antibodies to two adjacent regions located at the amino terminus of hepatitis C virus E2 glycoprotein. J Virol. 2013;87(1):37-51.

27. Keck ZY, et al. Antibody response to hypervariable region 1 interferes with broadly neutralizing antibodies to hepatitis $\mathrm{C}$ virus. J Virol. 2016;90(6):3112-3122.

28. Bailey JR, et al. Naturally selected hepatitis $\mathrm{C}$ virus polymorphisms confer broad neutralizing antibody resistance. J Clin Invest. 2015;125(1):437-447.

29. Munshaw S, et al. Computational reconstruction of Bole1a, a representative synthetic hepatitis $\mathrm{C}$ virus subtype 1a genome. J Virol. 2012;86(10):5915-5921.

30. Kinchen VJ, Bailey JR. Defining breadth of hepatitis $\mathrm{C}$ virus neutralization. Front Immunol. 2018;9:1703.

31. Georgiev IS, et al. Delineating antibody recognition in polyclonal sera from patterns of HIV-1 isolate neutralization. Science. 2013;340(6133):751-756.

32. Giang $E$, et al. Human broadly neutralizing antibodies to the envelope glycoprotein complex of hepatitis C virus. Proc Natl Acad Sci U S A. 2012;109(16):6205-6210.

33. Owsianka AM, et al. Broadly neutralizing human monoclonal antibodies to the hepatitis $\mathrm{C}$ virus $\mathrm{E} 2$ glycoprotein. J Gen Virol. 2008;89(pt 3):653-659.

34. Kinchen VJ, Cox AL, Bailey JR. Can broadly neutralizing monoclonal antibodies lead to a hepatitis $\mathrm{C}$ virus vaccine? Trends Microbiol. 2018;26(10):854-864

35. Tzarum N, et al. Genetic and structural insights into broad neutralization of hepatitis $\mathrm{C}$ virus by human $\mathrm{V}_{\mathrm{H}} 1-69$ antibodies. Sci $\mathrm{Adv}$. 2019;5(1):eaav1882.

36. Gopal R, et al. Probing the antigenicity of hepatitis $C$ virus envelope glycoprotein complex by high-throughput mutagenesis. PLoS Pathog. 2017;13(12):e1006735.

37. Pierce BG, et al. Global mapping of antibody recognition of the hepatitis $\mathrm{C}$ virus $\mathrm{E} 2$ glycoprotein: implications for vaccine design. Proc Natl Acad Sci U S A. 2016;113(45):E6946-E6954.
38. El-Diwany R, et al. Extra-epitopic hepatitis $\mathrm{C}$ virus polymorphisms confer resistance to broadly neutralizing antibodies by modulating binding to scavenger receptor B1. PLoS Pathog. 2017;13(2):e1006235.

39. Wasilewski LN, et al. A hepatitis C virus envelope polymorphism confers resistance to neutralization by polyclonal sera and broadly neutralizing monoclonal antibodies. J Virol. 2016;90(7):3773-3782.

40. Prentoe J, et al. Hypervariable region 1 and $\mathrm{N}$-linked glycans of hepatitis $\mathrm{C}$ regulate virion neutralization by modulating envelope conformations. Proc Natl Acad Sci U S A. 2019;116(20):10039-10047.

41. Mankowski MC, et al. Synergistic anti-HCV broadly neutralizing human monoclonal antibodies with independent mechanisms. Proc Natl Acad Sci U S A. 2018;115(1):E82-E91.

42. Carlsen TH, et al. Breadth of neutralization and synergy of clinically relevant human monoclonal antibodies against HCV genotypes 1a, 1b, 2a, 2b, 2c, and 3a. Hepatology. 2014;60(5):1551-1562.

43. de Jong YP, et al. Broadly neutralizing antibodies abrogate established hepatitis $\mathrm{C}$ virus infection. Sci Transl Med. 2014;6(254):254ra129.

44. Frey SE, et al. Safety and immunogenicity of HCV E1E2 vaccine adjuvanted with MF59 administered to healthy adults. Vaccine. 2010;28(38):6367-6373.

45. Liang TJ. Current progress in development of hepatitis $\mathrm{C}$ virus vaccines. Nat Med. 2013;19(7):869-878.

46. Tobin GJ, et al. Deceptive imprinting and immune refocusing in vaccine design. Vaccine. 2008;26(49):6189-6199.

47. Chung AW, Alter G. Systems serology: profiling vaccine induced humoral immunity against HIV. Retrovirology. 2017;14(1):57.

48. Hadlock KG, et al. Human monoclonal antibodies that inhibit binding of hepatitis $\mathrm{C}$ virus E2 protein to CD81 and recognize conserved conformational epitopes. J Virol. 2000;74(22):10407-10416.

49. Hsu M, et al. Hepatitis C virus glycoproteins mediate $\mathrm{pH}$-dependent cell entry of pseudotyped retroviral particles. Proc Natl Acad Sci US A. 2003;100(12):7271-7276.

50. Logvinoff C, et al. Neutralizing antibody response during acute and chronic hepatitis C virus infection. Proc Natl Acad Sci U S A. 2004;101(27):10149-10154.

51. Wasilewski LN, Ray SC, Bailey JR. Hepatitis $\mathrm{C}$ virus resistance to broadly neutralizing antibodies measured using replicationcompetent virus and pseudoparticles. J Gen Virol. 2016;97(11):2883-2893.

52. Bailey JR, Urbanowicz RA, Ball JK, Law M, Foung SKH. Standardized method for the study of antibody neutralization of HCV pseudoparticles (HCVpp). Methods Mol Biol. 2019;1911:441-450. 